\title{
Management Strategies and Clinicopathological Evaluation of Liver Abscess in the Tribal Area: A Cross Sectional Study
}

\author{
Ravindra K Kulkarni ${ }^{\circ}$ \\ Associate Professor, Department of Surgery, Zydus Medical College and Hospital, Dahod, Gujarat, India.
}

\section{Abstract}

Background: Liver abscess continues to be disease with considerable mortality in our country especially in Tribal Area. Locally made alcoholic drinks like neera, arrack may be the routes of faeco-oral transmission of amoebic cysts. Hence the aim of the study was to study the influence of alcohol, diabetics \& immunocompromised diseases leading to increased incidence of Liver abscess and to evaluate efficacy associated with most effective treatment. Subjects and Methods: The present study included 50 patients diagnosed with liver abscess. All the patients were under the treatment for the period of years. All cases of liver abscess diagnosed clinically and/or ultrasonographically, all cases of bacterial and parasitic liver abscess and the cases in evolving, liquefied \& ruptured stage with or without peritonitis were included in the study. Results: When the management part of the liver abscess was analysed 14 patients had abscess that was less than $200 \mathrm{cc}$ or had multiple small abscess involving both lobes that were managed conservatively. Total of 36 patients who had abscess $>200$ cc or left lobe abscess were subjected to intervention, of which 24 patients were treated with percutaneous aspiration with antibiotic coverage, 6 patients were treated with laparotomy procedure and 4 patients treated with drainage with laparoscope and 2 patients underwent ICD insertion. Conclusion: Liver abscess is a common condition in tribal area of India. It is still, a disease associated with considerable morbidity. Intraperitoneal rupture, pleural rupture, septicemia are the conditions that can occur. Multiple small liver abscess and solitary liver abscess $<200$ cc or size $<5 \mathrm{cms}$ can be successfully managed with conservative line of management with antimicrobials / antiamoebic drugs, but recurrences were noted. Percutaneous needle aspiration as well as Pigtail Catheter insertion is safe and highly effective method of managing liver abscess cases. Laparotomy and Drainage or Laparoscopic Drainage remains the standard of care for ruptured liver abscess.

Keywords: Alcohol, Amoebic serology, Liver abscess, Treatment

Corresponding Author: Ravindra K Kulkarni, Associate Professor, Department of Surgery, Zydus Medical College and Hospital, Dahod, Gujarat, India.

E-mail: lionravindrakulkarni@yahoo.com

Received: 06 April 2020

Revised: 11 May 2020

Accepted: 19 May 2020

Published: 27 May 2020

\section{Introduction}

Liver abscess (LA) is defined as collection of purulent material in liver parenchyma which can be due to bacterial, parasitic, fungal, or mixed infection. It is a common condition across the globe. ${ }^{[1]}$ Out of total incidence of LA, approximately two-thirds of cases in developing countries are of amoebic aetiology and three-fourths of cases in developed countries are pyogenic. ${ }^{[2]}$

Liver abscess are associated with mortality of up to $20 \%$ and are categorized into various types based on etiology, of which amoebic (ALA) and pyogenic (PLA) liver abscess are major types. ${ }^{[3]}$ Interestingly, ALA is more common in the developing nations. PLA constitutes the bulk of hepatic abscesses in developed nations. PLA result from ascending biliary tract infection, hematogenous spread through portal venous system, septicemia with involvement of liver by way of hepatic arterial circulation and secondary spread from intraperitoneal infection. ${ }^{[4]}$

A recent study in the US reported a national mortality rate of $6 \%$. Other recent series report a similar worldwide increase incidence and mortality rates between $11-31 \%$. Liver abscess is a common condition in India. India has 2 nd highest incidence of liver abscess in the world. ${ }^{[5]}$

Although no distinct clinical criteria exist for distinguishing ALA and PLA, the differential diagnosis can be made based on the following criteria- younger age, resident, or recent travel to areas of endemic amoebiasis, diarrhea, and marked abdominal pain raise clinical suspicion of ALA. ${ }^{[6]}$ The diagnosis is confirmed by ultrasonography (USG), serological tests such as indirect hemagglutination test, reddish brown (anchovy paste like material) aspirate from the abscess, negative gram stain, 
rapid resolution after metronidazole treatment. The diagnosis of PLA is based on picket fence configuration of temperature chart, nausea, vomiting, anorexia, hematological analysis of leukocytosis, anemia, and positive blood or aspirate culture for bacterial etiology. ${ }^{[6]}$

Liver abscesses are caused by bacterial, parasitic or fungal infection. Pyogenic abscesses account for three quarters of hepatic abscess in developed countries. ${ }^{[7]}$ While amoebic liver abscess causes two third of liver abscess in developing countries. Amoebiasis is presently the third most common cause of death from parasitic disease. ${ }^{[8]}$ Liver abscess continues to be disease with considerable mortality in our country. Locally made alcoholic drinks like neera, attack may be the routes of faeco-oral transmission of amoebic cysts. ${ }^{[9]}$ Hence the aim of the research was to study the influence of alcohol, diabetics \& immunocompromised diseases leading to increased incidence of Liver abscess and to evaluate efficacy, duration of hospital stay associated with most effective treatment.

\section{Subjects and Methods}

The present study included 50 patients diagnose with liver abscess. All the patients were under the treatment for the period of years.

All cases of liver abscess diagnosed clinically and/or ultrasonographically, all cases of bacterial and parasitic liver abscess and the cases in evolving, liquefied \& ruptured stage with or without peritonitis were included in the study. All the patient with age with greater than $18 \mathrm{yrs}$, patient with presence of traumatic liver abscess and patient with past history of liver abscess were excluded from the study.

Patient data was collected from all patients attending Hospital General Surgery OPD, Zydus

Medical College, Dahod, irrespective of their age/gender/ background /socio economic status. Dahod is District city in Gujarat, situated on the bank of Dudhimati river. The name also signifies two boundaries as borders of Rajasthan and Madhya Preadesh are nearby. It is Trible District of Gujarat and Trible area of Rajasthan and Madhya Pradesh are on boundaries. Zydus Medical College and Hospital is the only Tertiary Care Hospital available in this area. The patients were evaluated and followed up according to the protocol. Detailed history of patient was recorded in proforma. Patient was put on conservative line of management. Patient was followed up daily clinically and LFT \& USG Abdomen was repeated on the 3rd day if patient symptomatically not relieved. Repeat Ultrasound / CT /MRI Abdomen \& pelvis will be done immediately if patient condition does not improve/worsens or after 3-4 days as a routine. If the patient develops any of the complications like ruptured liver abscess into any of the serosal cavity, patient was immediately taken up for surgery. Pus was sent for gram's stain and culture and sensitivity. Anaerobic cultures were not done as the facility was not available in our hospital. Blood cultures were not routinely performed in all cases.

Patient was informed about any surgical procedure and consent taken. Age, gender, complaints, past-surgical history, past history of liver abscess, history of alcoholism, diabetes, any immunodeficiency states, any history of biliary tract disorder ,history of amoebic dysentery, jaundice was taken. Patient was examined in detail. Blood investigations and Xrays $\&$ and other radiological modalities performed was added. Complications if developed will be assessed in detail and management of the same and the further complications will be followed up.

Percutaneous needle aspiration was done on patients who had abscesses $>200 \mathrm{CC}$ with a $16 \mathrm{G} / 18 \mathrm{G}$ spinal needle. The site, depth, direction of aspiration was marked by ultrasonography. All patients were administered antibiotics intravenously initially upon admission. All patients were started with metronidazole at a dose of $40 \mathrm{mg} / \mathrm{kg} / \mathrm{day}$ is divided doses for 14 days. If upon aspiration, pus revealed growth of organisms than appropriate antibiotics were instituted in full course. Patients were examined daily for clinical improvement.

Improvement of pain, fever, anorexia, hepatomegaly were considered as a criterion for successful treatment. Laparotomy was considered in cases of suspected rupture of liver abscess with peritonitis. Ultrasonography was done as indicated. Relapses were noted and repeat aspirations were performed when necessary. Cure was defined as improvement clinically with subsidence of fever, and local signs, symptoms, decrease in WBC count and follow-up ultrasonography showed reduction in size $<3 \mathrm{~cm}$ in diameter and no evidence of relapses.

\section{Results}

The present study includes 50 patients diagnosed with liver abscess. The age range of the patients included in the study varied from $20-70$ years. The mean age was found to be 46 years. The maximum number of patients belongs to the age group $31-40$ years. Majority of the patients were male in the study.

In the present study the acute symptoms were seen in less than 7 days in majority of the patients, while in rest of the patients the duration for the symptoms to appear took 2 months. Owing to the clinical symptoms the abdominal pain was present in all cases. Symptom of diarrhoea was found in 8 patients. Jaundice was present in 12 patients. The respiratory symptom like cough was present in 15 patients. Hepatomegaly was present in $50 \%$ of cases that is 25 patients. Blood report of the patients 
showed that majority of the patient that is 36 patients had elevated WBC more than 11000 counts. Anaemic symptoms were found in 12 patients. Majority of the patients had avg $\mathrm{Hb}$ as $10.23 \mathrm{gm} / \mathrm{dl}$. Leucocytosis was found in 36 patients.

\begin{tabular}{ll}
\hline \multicolumn{2}{l}{ Table 1: Clinical signs present in the patients } \\
\hline Clinical Signs & Number of patients \\
\hline Abdominal tenderness & 50 \\
Diarrhoea & 8 \\
\hline Jaundice & 12 \\
Respiratory findings & 15 \\
Hepatomegaly & 25 \\
Fever & 45 \\
\hline
\end{tabular}

\begin{tabular}{|ll|}
\hline \multicolumn{2}{|c|}{ Table 2: Liver function test analysis } \\
\hline Liver function test & No. of patients \\
\hline S. albumin $(<3 \mathrm{mg} / \mathrm{dl})$ & 42 \\
ALP $(>150 \mathrm{IU} / \mathrm{L})$ & 40 \\
SGOT & 23 \\
SGPT & 21 \\
\hline Hypoalbuminaemia & 42 \\
\hline S. Bilirubin & 11 \\
\hline
\end{tabular}

When the liver function test analysis was done in all the 50 patients the serum albumin was found to be $<3 \mathrm{mg} / \mathrm{dl}$ in 42 patients and 40 patients had ALP $>150 \mathrm{IU} / \mathrm{L}$ and both the values were found to be statistically significant. Increase in the serum bilirubin was found in 11 patients, result was found to be $>2.4 \mathrm{gm} / \mathrm{dl}$. Alkaline phosphatise was found to be increase in 40 patients, hypoalbuminaemia was found in 42 cases, increased SGPT and SGOT was seen in 23 patients and 21 patients respectively.

When the management part of the liver abscess was analysed 14 patients had abscess that was less than $200 \mathrm{cc}$ or had multiple small abscess involving both lobes that were managed conservatively. Total of 36 patients who had abscess $>200 \mathrm{cc}$ or left lobe abscess were subjected to intervention, of which 24 patients were treated with percutaneous aspiration with antibiotic coverage, 6 patients were treated with laparotomy procedure and 4 patients treated with drainage with laproscope and 2 patients underwent ICD insertion.

\section{Discussion}

Amoebic liver abscess (ALA) is an important cause of inflammatory spaceoccupying lesions of the liver in the tropics. With the availability of potent anti-amoebic drugs relatively benign course with low mortality. Alcohol continues

\begin{tabular}{ll}
\hline \multicolumn{2}{|c|}{ Table 3: Treatment of protocol in the liver abscess patients } \\
\hline $\begin{array}{ll}\text { Trreatment Protocol } \\
\text { Conservative treatment } \\
\text { (Antibiotic coverage) }\end{array}$ & 14 \\
\hline Percutaneous aspiration & 24 \\
Laprotomy procedure & 6 \\
Drainage with laproscope & 4 \\
ICD insertion & 2 \\
\hline
\end{tabular}

to be an important risk factor for amoebic liver abscess. There is increased frequency of amoebic liver abscess in these alcoholics. The association between amoebic liver abscess and alcohol is well known with alcoholics having three times increased risk than that of non-alcoholic healthy control.

India being a tropical country and home to 400 million people harbouring E. histolytica, the causative organism of amoebic liver abscess, it assumes immense importance for thorough understanding of the same. ${ }^{[9]}$ The rising incidence in alcoholics \& immunocompromised individual has become a matter of grave concern as complications rate are high especially in this sub-group leading to increased morbidity and mortality. ${ }^{[10,11]}$

The prognostic markers were studied in amoebic liver abscess. Poor prognostic markers in amoebic liver abscess are much more common in alcoholics, HIV individuals. Liver abscess is a common condition in India. India has 2 nd highest incidence of liver abscess in the world. ${ }^{[12]}$ Liver abscesses are caused by bacterial, parasitic or fungai infection. Pyogenic abscesses account for three quarters of hepatic abscess in developed countries. While amoebic liver abscess cause two third of liver abscess in developing countries. ${ }^{[8]}$

Tenderness (100.0\%), Fever (94.0\%), Hepatomegaly (50.0\%) was common presentation in our series and was comparable to the studies listed below but Jaundice $(24.0 \%)$ was more common clinical presentation compared to study done by Hyo Min Yoo et al (7.0\%). Alcoholism was found to be the most consistent etiological factor in this study of liver abscess. 84/100 (84\%) of the cases of this study were found to be alcoholics as compared to other study by Shyam Mathur et al where $70 \%$ of the cases were alcoholic which concludes Alcoholism has a strong association with liver abscess patients.

Controversies in the management of liver abscess still exist. Surgical drainage of liver abscess has been an accepted therapy for decades. The diagnosis and treatment of liver abscess has changed due to advances in imaging techniques. Out of the 50 cases in this study, 14 patients who had multiple small abscess and solitary abscess with volume $<200 \mathrm{cc}$ or $<5 \mathrm{cms}$ size were treated conservatively. 36 patients who had abscess $>200 \mathrm{cc}$ or 
left lobe abscesses were subjected to Intervention as compared to Hyo Min Yo et al Study where $100.0 \%$ patients underwent intervention. ${ }^{[13]}$

Laparotomy as initial line of treatment was performed in 6 ruptured liver abscess cases and Laparoscopy drainage in 4 ruptured liver abscesses as compared to Hyo Min Yo et al Study where $21.0 \%$ patients underwent surgical intervention. ${ }^{[13,14]}$ In our Study Intercostal drainage was required in two cases in which the abscess had ruptured into pleural cavity, Patients survived. Thus in majority of cases percutaneous aspiration was the main form of treatment. All patients were started on antibiotics which were continued for 10- 14 days depending on improvement. Majority of patients responded excellently to percutaneous aspiration and antimicrobials. While patients who had smaller abscesses or multiple small abscesses were successfully managed with antimicrobial therapy alone but relapse rates were high.

\section{Conclusion}

Liver abscess is a common condition in India. It is still, a disease associated with considerable morbidity. Intraperitoneal rupture, pleural rupture, septicemia are the conditions that can occur. Multiple small liver abscess and solitary liver abscess $<200 \mathrm{cc}$ or size $<5 \mathrm{cms}$ can be successfully managed with conservative line of management with antimicrobials / antiamoebic drugs, but recurrences were noted. Percutaneous needle aspiration as well as Pigtail Catheter insertion is safe and highly effective method of managing liver abscess cases. Laparotomy and Drainage or Laparoscopic Drainage remains the standard of care for ruptured liver abscess.

\section{References}

1. Choudhary S. Evaluation of Various Treatment Modalities in Treating Diarrhoea Patient's at a Tertiary Care Centre: A Comparative Analysis. Asian J Med Res. 2019;8(4):SG01SG02. doi:10.21276/ajmr.2020.8.4.SG1.

2. Ghosh S, Sharma S, Gadpayle AK, Gupta HK, Mahajan RK, Sahoo R, et al. Clinical, Laboratory, and Management Profile in Patients of Liver Abscess from Northern India. J Trop Med. 2014;2014:1-8. Available from: https://dx.doi.org/10.1155/ 2014/142382. doi:10.1155/2014/142382.

3. Khan R, Hamid S, Abid S, Jafri W, Abbas Z, Islam M, et al. Predictive factors for early aspiration in liver abscess. World $\mathrm{J}$ Gastroenterol. 2008;14(13):2089-2089. Available from: https: //dx.doi.org/10.3748/wjg.14.2089. doi:10.3748/wjg.14.2089.

4. McDonald AP, Howard RJ. Pyogenic liver abscess. World J Surg. 1980;4(4):369-376. Available from: https://dx.doi.org/ 10.1007/bf02393151. doi:10.1007/bf02393151.
5. Currie BJ, Ward L, Cheng AC. The Epidemiology and Clinical Spectrum of Melioidosis: 540 Cases from the 20 Year Darwin Prospective Study. PLoS Neglected Trop Dis. 2010;4(11):e900-e900. Available from: $\quad$ https://dx.doi.org/10.1371/journal.pntd.0000900. doi:10.1371/journal.pntd.0000900.

6. Ratnottar J, Chauhan H, Modh F. Clinicopathological study and management of liver abscess in tertiary care center. Int Surg J. 2019;6(4):1332-1332. Available from: https://dx.doi.org/10.18203/2349-2902.isj20191272. doi:10.18203/2349-2902.isj20191272.

7. Okano H, Shiraki K, Inoue H, Kawakita T, Yamamoto N, Deguchi M. Clinicopathological analysis of liver abscess in Japan. Int J Mol Med. 2002;10:627-657.

8. Krige JEJ. ABC of diseases of liver, pancreas, and biliary system: Liver abscesses and hydatid disease. BMJ. 2001;322(7285):537-540. Available from: https://dx.doi.org/ 10.1136/bmj.322.7285.537. doi:10.1136/bmj.322.7285.537.

9. Sreedhar S, Nynasindhu A, Sahaja S. A Clinico-Pathological Study of Liver Abscess. J Evo Med Dent Sci. 2018;7(11):13761379. Available from: https://dx.doi.org/10.14260/jemds/2018/ 313. doi:10.14260/jemds/2018/313.

10. Trivedi G, Mishra S. To Assess the Efficacy of Postoperative Antibiotics after Appendectomy in Patients with NonPerforated Appendicitis. Asian J Med Res. 2019;8(4):SG10SG12. doi:10.21276/ajmr.2020.8.4.SG4.

11. Crane M, Iser D, Lewin SR. Human immunodeficiency virus infection and the liver. World J hepatol. 2012;4:91-96.

12. Sharma M, Dasarathy S, Verma N, Saksena S, Shukla DK. Prognostic markers in amebic liver abscess: a prospective study. Am J Gastroenterol. 1996;91:189-93.

13. Satish KR, Madhushankar L, Nataraj NR, Ramalingeshwara K, Laxmikantha L, Gupta MA. Hemanth V: Treatment strategies in liver abscess our experience. J Evol Med Dent Sci. 2013;2:8768-76.

14. Singh DV. Inguinal Hernia Mesh Repairs: Plug and Patch Versus Lichtenstein Mesh Repair Technique: A Teaching Hospital Based Study. Asian J Med Res. 2019;8(2):SG15SG20. doi:10.21276/ajmr.2019.8.2.SG4.

Copyright: (C) the author(s), 2020. It is an open-access article distributed under the terms of the Creative Commons Attribution License (CC BY 4.0), which permits authors to retain ownership of the copyright for their content, and allow anyone to download, reuse, reprint, modify, distribute and/or copy the content as long as the original authors and source are cited.

How to cite this article: Kulkarni RK. Management Strategies and Clinicopathological Evaluation of Liver Abscess in the Tribal Area: A Cross Sectional Study. Acad. J Surg. 2020;3(1):103-106.

DOI: dx.doi.org/10.47008/ajs/2020.3.1.22

Source of Support: Nil, Conflict of Interest: None declared. 